Revue internationale P.M.E.

Économie et gestion de la petite et moyenne entreprise

\title{
Les relations industrie-services dans la tertiarisation des économies
}

\section{Claire Lichtenstein}

Volume 6, numéro 2, 1993

URI : https://id.erudit.org/iderudit/1008208ar

DOI : https://doi.org/10.7202/1008208ar

Aller au sommaire du numéro

Éditeur(s)

Presses de l’Université du Québec

ISSN

0776-5436 (imprimé)

1918-9699 (numérique)

Découvrir la revue

Citer cet article

Lichtenstein, C. (1993). Les relations industrie-services dans la tertiarisation des économies. Revue internationale P.M.E., 6(2), 9-33.

https://doi.org/10.7202/1008208ar

\section{Résumé de l'article}

La tertiarisation des économies a souvent été analysée comme un phénomène de désindustrialisation devant mener, à terme, à une économie de services. L'analyse des limites de cette approche permet de contester l'idée d'une dissociation stricte entre secteur industriel et secteur des services.

En réalité, ces deux secteurs sont fortement connectés et l'une des caractéristiques majeures de l'évolution des économies développées est plus précisément la croissance des services à l'industrie, ce qui permet de soutenir l’idée que les économies avancées restent des économies de biens. 


\title{
Les relations industrie-services dans la tertiarisation des économies
}

\author{
Claire LICHTENSTEIN \\ Université de Nice - Sophia Antipolis*
}

\begin{abstract}
RÉSUMÉ
La tertiarisation des économies a souvent été analysée comme un phénomène de désindustrialisation devant mener, à terme, à une économie de services. L'analyse des limites de cette approche permet de contester l'idée d'une dissociation stricte entre secteur industriel et secteur des services.

En réalité, ces deux secteurs sont fortement connectés et l'une des caractéristiques majeures de l'évolution des économies développées est plus précisément la croissance des services à l'industrie, ce qui permet de soutenir l'idée que les économies avancées restent des économies de biens.
\end{abstract}

\section{ABSTRACT}

The tertiarisation of economies has been often analysed as a phenomenon of de-industrialization which could lead to a "service economy." By pointing out the limits of this approach, we can then contest the idea of a strict dissociation between industry sector and services sector.

In reality, these two sectors are heavily connected and the main characteristic of the developed economies evolution is more precisely the growth of producer services. Owing to this findings, we defend the notion that the developed economies remain "commodity economies."

\section{RESUMEN}

La terciarisación de las económias ha sido a munudo analisada como un fenomeno de desindustrialización que debe llevar a cabo una económia de servicio. El análisis de los limites de esta mirada permite contestar la idea de una disociación estrica entre el sector industrial y el sector de los servicios.

- Claire Lichtenstein, née Lescarcelle-Evin, détient un doctorat de $3^{\mathrm{e}}$ cycle en Économie du travail et des ressources humaines. Ses intérêts de recherche portent actuellement sur les échanges internationaux de services. Elle est aussi chercheure au LATAPSES, à l'Unité mixte du CNRS. Adresse : LATAPSES, Groupe des laboratoires du CNRS, 250, rue A. Einstein, Sophia Antipolis 1, 06560 Valbonne, France. 
En realidad, esos dos sectores están conectados, y unas de la características mayores de la evolución de las económias desarolladas es más precisamente el cricimiento de los servicios de la industria, lo que permite sostener la idea que las económias desarolladas siguen siendo económias de bienes.

\section{Introduction}

Les difficultés d'analyse du phénomène de «tertiarisation » des économies développées proviennent de la délicate caractérisation de ce que l'on appelle généralement «le tertiaire». En effet, ce dernier n'apparaît pas comme un secteur au sens strict du terme, mais plutôt comme un ensemble d'activités hétérogènes. Il comprend une variété d'activités économiques nécessitant un investissement plus ou moins important en capital humain, un capital technique plus ou moins élevé, parfois même inexistant, ainsi que des activités étroitement liées au marché privé ou des activités publiques avec des considérations marchandes limitées (Kuznets, 1966).

Le fondement commun de ces activités est de ne pas produire de biens matériels, mais de fournir un produit «intangible», difficilement mesurable (Aanestad, 1987). Le critère de matérialité a cependant des limites : non seulement plusieurs services sont associés à un produit physique, mais dans certains cas, une partie du service se reflète dans l'apparence d'un produit tangible. Aussi est-il difficile de considérer que tous les services n'ont aucune durabilité (Smith, 1972). Certaines analyses remettent en question une frontière stricte entre les biens et les services, puisque «l'homme ne peut pas créer de choses matérielles. Il peut produire de nouvelles idées ; mais quand il produit des choses matérielles, il ne produit réellement que des services » (Marshall, 1979). Le recours au critère de matérialité apparaît dans de nombreuses analyses pour aider à définir la notion de tertiaire; toutefois, il ne devra pas être retenu sans réserve et il conviendra par la suite de garder ses limites en mémoire. Outre ce caractère d'intangibilité, de nombreux auteurs s'accordent à reconnaître à ce secteur particulier un caractère résiduel : est tertiaire ce qui n'est ni primaire, ni secondaire, c'est-à-dire l'ensemble des branches qui produisent des services et non des biens matériels (Vernieres, 1985). Mais en fait, il n'existe pas de définition qui fasse autorité sur les limites de classification des activités de services. Par exemple, certaines études incluent les transports, les communications et les services publics ${ }^{1}$; d'autres n'incluent que les deux premiers, d'autres encore excluent les trois et les intègrent dans l'industrie (Stigler, 1956).

1. Tels que l'eau, le gaz, l'électricité et l'assainissement. 
Dans le courant des années 70, la tertiarisation des économies a souvent été analysée comme un phénomène de désindustrialisation devant mener à terme à une «économie de services». Ces analyses, fondées sur l'idée d'une croissance par étapes successives (Rostow, 1960), défendent la thèse d'une tendance historique inéluctable des économies développées vers une société postindustrielle (Touraine, 1969; Bell, 1973) où l'information jouerait un rôle privilégié en devenant la ressource centrale et une source de pouvoir à l'intérieur des organisations. Ces approches soulignent la tendance générale vers une réduction de l'emploi industriel en termes relatif et absolu (Cairncross, 1978). Cette réduction peut être la conséquence de l'effet d'une réduction relative de la consommation de produits industriels (Bacon et Eltis, 1976), des caractéristiques spécifiques des récentes innovations technologiques ou, encore, la conséquence de l'évincement du secteur industriel par le secteur public.

Dans cette perspective, le présent article peut être interprété comme une simple tentative d'évaluation de la validité des thèses de tertiarisation et de désindustrialisation. Pour ce faire, deux étapes seront nécessaires. La première reviendra sur le fondement même de ces thèses, à savoir la représentation du système économique comme articulation de trois secteurs : primaire, secondaire, tertiaire. C'est en effet ce découpage qui permet de penser l'évolution économique comme une suite de périodes caractéristiques, marquées par des décroissances relatives successives des secteurs primaire et secondaire. Dans cette optique, nous montrerons comment est née la représentation évoquée. En d'autres termes, nous renouerons avec la problématique d'auteurs dont les textes sont aujourd'hui plus invoqués qu'analysés et qui sont à l'origine du découpage sectoriel de l'économie. Cette étude nous permettra de mettre en évidence une représentation beaucoup plus fine et nuancée des secteurs et ainsi de relativiser le degré de généralité et la portée du découpage trisectoriel. Dans une deuxième étape, nous contesterons l'idée d'une dissociation stricte entre secteur industriel et secteur des services. En nous appuyant sur un certain nombre de travaux récents, nous chercherons à montrer que ces deux secteurs sont, en réalité, fortement connectés et que l'une des caractéristiques majeures de l'évolution des économies développées contemporaines est précisément la croissance des services à l'industrie. Sera ainsi démentie l'idée d'une tertiarisation et celle, corrélative, d'une désindustrialisation de ces économies.

Au total, loin d'être une économie de services purs, le type d'économie vers lequel nous tendons se caractériserait ainsi par une imbrication croissante entre industrie et services, c'est-à-dire entre production de biens et production de services. 


\section{Le fondement de la représentation trisectorielle du système économique}

Société tertiaire et tertiarisation sont deux expressions qui mettent en relief des formes particulières d'organisation économique et sociale. La terminologie «primaire, secondaire et tertiaire » remonte au début des années 30. C'est à partir du moment où la part des activités non agricoles et non industrielles va dépasser celle des activités industrielles qu'est élaborée la théorie des trois secteurs (Fisher, 1935). A.G.B. Fisher est le premier auteur -bien que la «paternité » en soit souvent conférée à $\mathrm{C}$. Clark-, à avoir dégagé trois étapes principales dans la société, à partir de l'observation des habitudes de dépenses des individus; cette observation lui permet de généraliser ce qui doit arriver quand des sociétés pauvres pensent pouvoir dépenser plus que ce qu'elles dépensaient auparavant. Partant de cette observation, il définit trois étapes qui successivement sont complémentaires par rapport au développement économique. L'étape tertiaire se situe au $\mathrm{xx}^{\mathrm{e}}$ siècle. C'est une époque où les problèmes de production manufacturière sont résolus. Il devient donc possible de détourner une proportion croissante de l'effort et du temps humain, ainsi que du capital, vers des productions qui ne sont ni primaires, ni secondaires. Ainsi, les activités économiques sont-elles divisées en trois catégories (Fisher, 1935 et 1945). La première catégorie comprend le travail agricole et minier qui a pour objet direct la production des aliments et des diverses matières premières. La seconde recouvre les industries de transformation sous toutes leurs formes. La troisième, enfin, est composée du reste, c'est-à-dire d'un vaste ensemble d'activités consacrées à la fourniture de «services » allant des transports au commerce, en passant par les loisirs, l'instruction, la création artistique et la philosophie. On constate alors que l'emploi et les investissements n'ont cessé de glisser des activités primaires essentielles et sans lesquelles la vie, même sous les formes les plus primitives, serait impossible, vers les activités secondaires et tertiaires. Le glissement de la main-d'œuvre vers les productions secondaire et tertiaire, révélé par les statistiques, est l'inéluctable conséquence du progrès économique: l'un ne va pas sans l'autre. Si la moyenne du revenu s'élève, une partie en sera dépensée à acquérir des objets différents de ceux achetés jusqu'alors. Or, pour acheter ces biens, il faut d'abord les produire sur une échelle adéquate; il faut que la main-d'œuvre se déplace: empêcher ce déplacement équivaut donc à empêcher l'amélioration du niveau moyen du revenu (Fisher, 1945).

Le terme tertiaire fut inventé avec l'objectif de la construction d'un schéma conceptuel qui pouvait aider à analyser des domaines de l'activité dans lesquels, à un moment donné, une croissance rapide devait étroitement s'harmo- 
niser avec les exigences du progrès économique. C'est pourquoi le premier axe d'analyse fut établi en termes de structure de la demande du consommateur. L'existence d'un degré élevé et significatif de corrélation entre le niveau du revenu national et la proportion de la population non engagée dans l'agriculture et l'industrie peut être acceptée comme un fait dont on ne peut douter (Fisher, 1946).

Un des grands intérêts des travaux de Fisher réside dans l'examen systématique des migrations professionnelles dans les trois secteurs de l'activité, le propos d'alors étant de déterminer les causes de l'insuffisante mobilité de la main-d'œuvre. La réponse est trouvée d'une part dans la vraie direction du développement économique et, d'autre part, dans la crainte, tant des nations que des individus, à l'égard de la nouveauté, du risque et de l'inconnu (Fisher, 1935 et 1945). Pour argumenter son propos, A.G.B. Fisher s'appuie sur le secteur agricole. Il remarque que l'on déplore partout le déracinement des masses paysannes et la désertion des campagnes ; ce n'est en fait que la conséquence naturelle du perfectionnement de l'agriculture. Dans le secteur industriel où le progrès technique engendre un accroissement plus marqué de la productivité, il faut s'attendre également à ce que la demande de main-d'œuvre diminue aussi à l'avenir, car il y a une limite de saturation dans la consommation des biens manufacturés. Ainsi, ce n'est pas en restreignant l'importance ni en protégeant artificiellement les industries menacées que l'on évitera le chômage (Fisher, 1935). La solution est le transfert calculé, organisé et contrôlé de la main-d'œuvre dans le seul secteur qui reste ouvert: le tertiaire. Ce dernier est le moins touché par le progrès technique, la machine n'y remplace l'homme que rarement et partiellement. Aussi, l'offre de «services » reste-t-elle souvent inférieure à la demande. Le progrès économique qui repose sur la croissance du savoir est susceptible d'entraîner des modifications considérables dans l'importance relative du capital comme facteur de production (Fisher, 1933). Il implique des changements et parfois même des désagréments et des souffrances. C'est pourquoi il existe une résistance au progrès. En ce sens, l'attitude de l'homme est paradoxale, car il veut profiter des avantages du progrès, tout en aspirant à la stabilité et à la sécurité (Fisher, 1935). Afin de modifier ces comportements, il faut trouver une théorie du changement en économie politique. Ces changements peuvent être observés par les effets qu'ils exercent sur l'offre et la demande de biens. Dans l'analyse des producteurs de biens tertiaires de cet auteur, on remarque la tendance de certains à mépriser et à taxer de parasitaires les activités professionnelles qui procurent à l'homme, non des biens de première et seconde nécessité, mais des loisirs, du délassement, le développement des aptitudes artistiques, intellectuelles et scientifiques, et on n'encourage pas l'expansion de ces activités professionnelles (Jaccard, 1944). Ainsi, dans tous les secteurs de la vie économique, on constate une résistance à l'adaptation. 
À travers cette analyse, c'est la résistance au progrès technique qui menace l'équilibre de toute la vie économique. Pour A.G.B. Fisher, cette résistance au progrès est l'une des causes de la persistance de la dépression économique. En effet, sa théorie suit de près la Grande Crise des années 30 . Cette coïncidence ne semble pas fortuite dans la mesure où il décompose les activités économiques en trois grands groupes ayant des caractéristiques de production et de marché différentes, pour tenter d'expliquer les crises. Ainsi, la prospérité d'un peuple dépend directement non seulement de la division plus ou moins grande de son travail ou de la proportion plus ou moins forte de ses travailleurs établis dans le secondaire et dans le tertiaire, mais surtout de l'aisance et de la rapidité avec lesquelles un peuple est susceptible de passer de la production primaire à la production secondaire puis tertiaire, sans se laisser retenir par les entraves de la crainte, de la routine et de l'égoïsme.

La diffusion du découpage sectoriel établi par A.G.B. Fisher a été relativement faible. Quand la production tertiaire a été proposée comme outil d'analyse économique, il n'y avait pas de précédent dans la littérature économique. Les termes de productions primaire et secondaire ont été utilisés à travers le monde dès le début des années 30, notamment en Australie et en NouvelleZélande $^{2}$, comme une expression commode pour résumer les activités différentes de celles des fermiers et des industriels. Mais en fait, il n'y avait pas de convention universelle pour dire que ces significations étaient appropriées à ces termes. Ces derniers n'étaient apparemment pas répandus aux États-Unis car, en 1938, un auteur américain trouva nécessaire d'expliquer à ses lecteurs que la production primaire comme synonyme «d'activités agricoles et élevage du bétail » était une habitude australienne (Fisher, 1952). Dans les faits, les idées de A.G.B. Fisher n'ont pas été accueillies avec autant de faveur qu'elles le méritaient par l'Angleterre des années 1935-1940. Pour cette raison sans doute, elles n'ont eu que peu d'échos dans le monde où peu d'auteurs ayant travaillé sur le découpage sectoriel et le progrès économique l'ont cité (Jaccard, 1953). Seul A. Sauvy s'y réfère lorsqu'il parle, en 1952, de la « révision étonnante des conceptions économiques en cours depuis quelques années » dans la Théorie générale de la population ${ }^{3}$.

Les analyses de A.G.B. Fisher, même si elles sont critiquables dans la mesure où elles ne présentent pas de véritable modèle de croissance, fournissent néanmoins une décomposition permettant d'appréhender de manière synthétique et qualitative des phénomènes qui ne sont pas, pour l'essentiel, réductibles au seul aspect quantitatif. Si l'on considère que l'organisation économique et

2. Pays où Fisher avait puisé ses observations.

3. Sauvy (1952), Théorie générale de la population, vol. 1, Paris, PUF, Économie et croissance. 
sociale ne peut être réduite à l'évolution dans le temps d'une série de trois secteurs, il n'en demeure pas moins que ces transformations, leurs effets et leurs tendances sont mis en évidence et que cela rend possible une analyse sur la signification socio-économique de la croissance comparée des secteurs et, par là-même, comme on le verra ultérieurement, une remise en cause du découpage sectoriel.

Depuis A.G.B. Fisher, la notion de secteur a évolué. C'est souvent à C. Clark (1940) que l'on attribue la décomposition de l'économie en trois secteurs d'activité et la découverte de la corrélation entre la prospérité d'un pays et la répartition des activités professionnelles dans ce pays. Or, comme nous venons de le voir, l'essentiel à ce propos a déjà été exposé par A.G.B. Fisher bien avant 1940, avec une parfaite clarté. C. Clark (1940) a jugé souhaitable de distinguer dans le processus économique trois grandes subdivisions dont on peut fixer les limites «en faisant appel au bon sens, tout en restant très précis ». Il ajoute que des différences fondamentales existent entre les lois économiques qui régissent ces trois secteurs. Dans le premier secteur, les activités dépendent toutes de l'utilisation directe et immédiate de ressources naturelles. Compte tenu de cette dimension, elles ne peuvent être mises en œuvre qu'à l'endroit où se trouvent ces ressources ; cette caractéristique constitue l'une des considérations les plus importantes qui les distingue de l'industrie. À ce stade du développement, C. Clark remarque que l'exploitation minière est un cas limite, pouvant être classée soit dans l'agriculture, soit dans l'industrie, mais qui mérite dans tous les cas d'être classée à part. Les traits communs à l'ensemble des activités agricoles résident dans leurs processus productifs assez lents. Malgré les possibilités qu'offre le progrès technique, les processus agricoles, à de rares exceptions près, subissent la loi des rendements décroissants ${ }^{4}$. Le secteur secondaire est défini comme regroupant les activités ayant un processus qui n'utilise pas directement les ressources naturelles et qui produit sur une grande échelle et de façon continue des biens transportables. Cette définition est intéressante dans la mesure où elle exclut la production de biens intransportables comme, par exemple, le bâtiment et les travaux publics, de même que les procédés discontinus sur une petite échelle, tels que la fabrication artisanale. Ainsi, la substance essentielle de l'industrie réside dans le fait que les matières premières peuvent être transportées sur de très longues distances, nécessiter des investissements lourds ainsi qu'un niveau élevé d'organisation et enfin suivre la loi des rendements croissants ${ }^{5}$. La définition de ces deux secteurs laisse donc supposer que

4. À noter que c'est essentiellement cet argument qui conduit Clark à classer la production minière dans le primaire.

5. Clark utilise l'argument des rendements croissants dans la première édition de son ouvrage (1940) et l'abandonne dans l'édition de 1951. 
le tertiaire inclut les activités restantes, c'est-à-dire le bâtiment et les travaux publics, les transports et les communications, le commerce et les finances, les services «professionnels ${ }^{6}$ », l'administration et les services personnels. La seule raison apparente pour laquelle $\mathrm{C}$. Clark place les transports et les communications dans le tertiaire est que ce sont des services (Wolfe, 1955). À ce niveau de l'analyse, il faut remarquer que C. Clark précise qu'il faut distinguer les services directement fournis à l'acquéreur définitif, que ce soit le consommateur, le capitaliste ou l'État, et les services utilisés pour mettre en œuvre d'autres processus de production, transports de biens, commerce de gros, services à des fins commerciales.

Dans le type d'analyse proposé par C. Clark, à mesure que le temps passe et que les communautés atteignent un stade plus avancé de développement économique, la main-d'œuvre agricole tend à décroître par rapport à la maind'œuvre industrielle qui, elle-même, tend à décroître par rapport aux effectifs employés dans les services. C. Clark se réfère alors à W. Petty (sans préciser la source) qui en 1691, se demandant pourquoi le revenu des Hollandais de son temps était plus élevé que celui des Anglais, avait trouvé l'explication dans le fait que, pour une plus forte part, les Hollandais vivaient de l'industrie et du commerce. C. Clark cite W. Petty: «il y a beaucoup plus à gagner par l'industrie que par l'agriculture et beaucoup plus par le commerce que par l'industrie. Nous pouvons constater qu'à mesure que le commerce et les "arts curieux" se développent, l'agriculture doit décliner, ou bien les salaires agricoles doivent augmenter et les rentes foncières diminuer en conséquence». W. Petty avait mis l'accent sur les «économies» importantes dont bénéficiaient les Hollandais, non seulement dans les transports, mais aussi dans l'administration et la fourniture de services «professionnels» en raison de leur étroite proximité les uns des autres et en l'absence de longues distances. Il lui apparut donc que le niveau plus élevé de développement économique était lié aux Pays-Bas, et devait par là même être lié ailleurs, à l'emploi d'une forte proportion de la population active dans l'industrie et le commerce plutôt que dans l'agriculture. Ces éléments permettent à C. Clark de démontrer la rigoureuse exactitude tant des prévisions que des constatations de W. Petty. De plus, la délimitation entre les trois secteurs apparaît clairement dans le passage cité. Ainsi, W. Petty montre le premier que la libération des contraintes de la vie primitive où seule comptait la nécessité de se nourrir, pour s'adonner aux arts et métiers, puis se vouer aux recherches de la vie intellectuelle, n'est pas un «luxe», c'est-à-dire un gaspillage ou du moins un appauvrissement dans le domaine économique mais, au contraire, un enrichissement matériel autant que moral et spirituel. Il semble même avoir deviné que les transferts de population active du premier au second, puis du

6. Ils correspondent à ce que l'auteur intitule les «professional services». 
second au troisième des différents secteurs étaient non seulement le signe visible, mais encore l'une des conditions les plus importantes du progrès humain (Jaccard, 1953). Dans son ouvrage, C. Clark qualifie les remarques de W. Petty de «loi de Petty» et explique que le degré de prospérité d'un pays est en relation directe avec la répartition de ses travailleurs dans les trois secteurs généraux de la vie économique. Plus la proportion est forte dans le secondaire et surtout dans le tertiaire, plus le progrès économique et social est manifeste. C. Clark a eu le mérite de donner à la « loi de Petty » une éclatante justification basée sur des études statistiques touchant de nombreux pays. En effet, on observe entre le niveau de revenu moyen et la part de la population active engagée dans le tertiaire un parallélisme frappant. Ainsi, ce ne peut être l'effet d'une simple coïncidence et l'importance du tertiaire apparaît non pas comme la conséquence, mais comme l'une des causes même de la richesse d'un pays (Jaccard, 1953). Le commerce, essentiellement de transport, représente pour de nombreux auteurs du XVII ${ }^{e}$ siècle l'activité essentielle (Dockes, 1969). Peu d'auteurs avaient utilisé les travaux de W. Petty. À la fin du XIX ${ }^{\mathrm{e}}$ siècle, seuls quelques auteurs (dont Giffen en 1887) ont relevé que la prospérité matérielle des nations industrialisées comme l'Angleterre éveille des besoins toujours croissants de biens et services non matériels, créant des possibilités d'emploi dans des domaines nouveaux (Jacard, 1953) ${ }^{7}$.

La critique générale que l'on peut faire d'emblée à propos des travaux de A.G.B. Fisher et C. Clark est qu'ils étendent l'argument du modèle de développement du primaire et du secondaire aux services (Katouzian, 1970). Dans leurs théories, l'accroissement et le déclin des parts des secteurs agricole et industriel s'expliquent généralement par la différence du taux de croissance de la productivité dans ces deux secteurs et par l'impact de la division du travail. En 1952, Fisher propose des critères plus précis en introduisant le concept de l'élasticité-revenu; les activités primaires étant définies comme celles dont les produits ont une élasticité inférieure à 0,5 , les activités secondaires, entre 0,5 et 1 et les tertiaires, supérieure à 1 . Toutefois, si les études empiriques ont mis ces critères en valeur pour le primaire et le secondaire, il n'en a pas été de même pour le tertiaire. En effet, l'élasticité de la demande par rapport au revenu, testée pour le cas des États-Unis, n'est pas significativement supérieure à l'unité (Fuchs, 1968). Ainsi, la définition du tertiaire par différence semble garantir l'exhaustivité de toute classification et, si les exigences d'une logique

7. Hobson (1894) dans The Evolution of Modern Capitalism (Londres, Walter Scott Ltd) avait également apporté des idées sur l'évolution des activités sans toutefois parler de secteurs. Il avait démontré le déclin relatif de l'agriculture et de certains aspects de l'industrie comparativement aux branches de la production non matérielle. De plus, son raisonnement s'appuyait sur la loi d'Engel. 
formelle et rigoureuse sont respectées, une définition de ce type est probablement la meilleure que l'on puisse faire pour la production tertiaire (Fisher, 1952). Cependant, il ne semble pas entièrement satisfaisant de traiter le tertiaire comme un groupe «fourre-tout» dans lequel chaque bien ne convenant pas ailleurs est classé. Si l'on reprend la classification selon une "hiérarchie des besoins ", conformément à l'extension qui a été faite à partir de la loi $\mathrm{d}^{\prime}$ Engel ${ }^{8}$, on peut assimiler le tertiaire à des biens de luxe. Sachant que la satisfaction des biens de luxe varie dans le temps en fonction des types de consommation et des niveaux de production, cela entraîne des variations dans la fixation des biens et services dans tel ou tel secteur.

Il y a également des limites importantes aux analyses de Fisher et de Clark qui basent leur raisonnement sur le fait qu'une part élevée de la population active dans le tertiaire est à la fois une conséquence et un indicateur d'un niveau de vie élevé. Cette base analytique de leur généralisation a été critiquée à plusieurs niveaux (Bauer et Yamey, 1951). En effet, une part substantielle des produits tertiaires ne sont pas des produits de luxe, avec une élasticité relativement élevée de la demande, et inversement, des produits primaires et secondaires peuvent être des produits de luxe. De plus, il peut très bien y avoir une substitution capital-travail à grande échelle dans la production tertiaire, dans le processus de progrès économique. Pour Bauer et Yamey, il n'y a pas de raison de croire que lorsque la richesse augmente, une plus grande part de biens de luxe consommés doit consister en des produits relevant d'activités tertiaires. La négligence de l'effet de substitution détruit la validité générale de la loi quantitative corrélant le revenu réel de la société et la proportion de population active dans le tertiaire. La production tertiaire est l'agrégation de nombreuses activités dissemblables; en conséquence, il n'y a pas de raison pour que la demande de chacune d'elle suive la même tendance. Le seul trait commun de toute la production tertiaire est que l'output est immatériel. Par conséquent, toute corrélation observée entre le progrès économique et la répartition professionnelle doit être considérée

8. Il faut faire remarquer que ce que l'on appelle communément la loi d'Engel ne concerne en fait que la dépense de nourriture (Zimmerman, 1932: «Ernst Engel's law of expenditures for food », Quartely Journal of Economics, décembre ; Houthakker, 1957: «An international comparison of household expenditure patterns, commemorating the centenary of Engel's law », Econometrica, vol. 25, $\mathrm{n}^{\circ}$ 4, octobre; Berthomieu, 1966 : «La loi et les travaux d'Engel», Consommation, $\mathrm{n}^{\circ} 4$, octobre-décembre). À ce propos, Berthomieu souligne que divers auteurs, souvent anglo-saxons, ont attribué à Engel la paternité de plusieurs lois qui seraient non seulement relatives aux dépenses de nourriture, mais aussi à celles de vêtements, d'habillement, de chauffage et d'éclairage... Ces inexactitudes sont très probablement dues à l'interprétation un peu large de l'étude d'Engel qu'en donna l'auteur américain Wright en 1876. 
davantage comme un accident statistique que comme un indicateur ou une preuve d'une loi économique significative.

On assiste donc, à travers certaines analyses, à la remise en cause des résultats et arguments de Fisher, tout d'abord, en rejetant la thèse de la corrélation entre le progrès économique et l'accroissement relatif des effectifs employés dans le tertiaire (Bauer et Yamey, 1951), mais aussi à une position plus radicale soutenant que les instruments disponibles pour analyser les problèmes de modifications et de mouvements professionnels ne nécessitent ni ne reçoivent probablement d'assistance de la classification de l'activité économique en «primaire, secondaire et tertiaire» (Bauer et Yamey, 1954). La réponse apportée à cette critique (Fisher, 1954) consiste en la précision du rôle beaucoup plus modeste conféré à la production tertiaire, qui n'est en fait suggérée que comme une simple classification et qui ne prétend pas représenter un substitut partiel ou total des instruments standard de l'analyse économique.

Dans le prolongement des analyses sectorielles, d'autres types de travaux créent un élément nouveau: le découpage sectoriel doit s'appuyer sur le progrès technique (Fourastie, 1950). Ainsi, les définitions précédentes apparaissent comme des énumérations purement formelles. C'est un choix alternatif qui est pris, basé sur le degré d'intensité du progrès technique dans les diverses activités. Le primaire se trouve caractérisé par un progrès technique modéré, le secondaire, par un progrès technique plus rapide et le tertiaire, par un progrès technique négligeable, voire inexistant. De manière générale, un critère sûr pour reconnaître le tertiaire repose sur l'identité du rendement du travail dans l'espace. En effet, la constance dans le temps entraîne une constance dans l'espace. Telle est la propriété fondamentale du tertiaire : le rendement du travail y est le même dans tous les pays. L'utilisation du progrès technique a pour objectif d'introduire des critères d'homogénéité dans le secteur tertiaire, considéré jusqu'ici comme résiduel et fortement hétérogène. Néanmoins, ce nouveau critère, en voulant apporter une dimension plus rigoureuse, dénature l'idée primitive de Fisher, puis celle de Clark, en lui donnant une configuration plus mouvante ainsi qu'un caractère plus superficiel, alors que leur objectif était de poser une commodité d'analyse, qui par ailleurs a été fort utile pour les travaux ultérieurs. Bien que la composition des secteurs soit variable dans le temps, dans la réalité, on observe une certaine stabilité et une certaine coïncidence avec les classifications précédentes de Fisher et de Clark, même si la théorie de Fourastié diverge fondamentalement d'un point de vue doctrinal. Eṇ effet, selon l'évolution du progrès technique, on trouvera les transports et les communications dans le secondaire, puis dans le tertiaire. Pour Fourastié, les transports sont à la limite des deux secteurs ; par contre, les communications appartiennent au tertiaire. Ces deux activités présentent un caractère mixte ; elles sont typiquement «indociles » et confirment l'idée toujours présentée par l'auteur du caractère capricieux du 
progrès technique. En revanche, il y a un tertiaire stable composé du commerce de détail, des professions libérales et des administrations.

À travers ces différentes analyses, on s'aperçoit que ni Clark, ni Fourastié n'ont accepté le raisonnement de Fisher, et Clark, après avoir forgé sa démonstration sur la loi des rendements croissants et décroissants (première édition, 1940) qu'il abandonne dans la deuxième édition de 1951, isole le tertiaire en le considérant comme «ce qui reste» et en le limitant aux activités produisant des «biens intangibles ${ }^{9}$ ». Cependant, certaines phrases indiquent qu'il aurait admis que les termes étaient convenables, mais arbitraires (Wolfe, 1955). Clark regroupe ainsi toutes les activités produisant un output non matériel comme secteur de l'économie. Quant à Fourastié, son argument réside dans le rôle fondamental joué par le progrès technique et il reproche à la division de Clark d'être trop énumérative. L'approche de Fisher, basée sur la structure de la demande du consommateur, reconstitue presque une identité entre «primaire, secondaire, tertiaire e et «biens les plus essentiels, moins essentiels et les moins essentiels ». Dans cette approche, le secteur primaire ne pose pas de problème, car il inclut en majörité la production et la distribution de biens essentiels. Mais concernant le secondaire, bien que produisant modérément des biens essentiels, il est ultérieurement défini comme étant limité à l'industrie. Par conséquent, si la demande de consommation tertiaire semble désigner des biens de luxe ou semi-luxe, Fisher insiste néanmoins sur le fait que tous les services qui permettent la satisfaction directe du consommateur sont dans le tertiaire qui inclut alors des services relativement essentiels, comme les services médicaux. Donc, si l'on adopte les termes introduits par Fisher et utilisés par Clark, on peut prétendre que les conditions du progrès économique incluent le détournement de la population active des occupations primaires vers les secondaires et, particulièrement, vers la production tertiaire (Ojala, 1952; Triantis, 1953). Ceci étant, il n'y pas dans leurs analyses de mention explicite d'un mécanisme de croissance qui expliquerait le passage d'une étape à l'autre (Petit, 1986).

Comme cela a déjà été mentionné, le point faible de leur théorie semble résider dans l'extension de l'argument du modèle de développement du primaire et du secondaire pour les services. Le tertiaire semble moins apte à être un modèle de 'généralisation d'ensemble, en raison de son mode de développement (Katouzian, 1970). La réalité historique et contemporaine démontre qu'un déclin de l'emploi agricole n'est pas toujours accompagné d'une croissance des activités secondaires. Il peut en résulter un accroissement direct de l'emploi tertiaire; de plus, bien qu'il y ait une relation directe pour chaque pays

9. On peut d'ailleurs faire remarquer que Clark signale que l'expression «activités tertiaires » semble avoir atteint des limites et pourrait être remplacée par l'expression « activités de services ». 
étudié par Clark entre le niveau de revenu par tête et la part de l'emploi tertiaire, cette relation est beaucoup plus faible lorsque l'on compare les pays (Singelmann, 1978).

Ainsi, la théorie des trois secteurs liée à celle des étapes de la croissance économique (Rostow, 1960) renvoie à l'idée d'une hiérarchisation des besoins conformément à l'extension de la loi d'Engel. On s'aperçoit que ce sont des analyses très agrégées des activités; elles considèrent le tertiaire comme un ensemble pouvant être analysé comme un secteur. Toutes ces analyses ont en commun d'opposer le tertiaire à l'industrie. Si l'on définit le tertiaire selon le critère voulant qu'il soit un ensemble résiduel, on s'aperçoit que le tertiaire ne correspond pas dans la réalité à la définition d'un secteur, d'une industrie ou d'une branche (Siniscalco, 1985). Il correspond en fait à un ensemble artificiel d'activités hétérogènes du point de vue de l'offre et de la demande, mais aussi des coûts, de la productivité, des parts de marché, de l'avancée technologique, de l'intégration dans le reste du système productif, etc. Il faut également noter que les différentes analyses de la croissance de l'emploi tertiaire la limitent à des modifications endogènes au secteur considéré comme une entité (comme par exemple le progrès technique ou la productivité) ou au plus à l'influence de facteurs exogènes communs à l'ensemble des secteurs (comme par exemple la politique sociale de l'État). Dans les deux cas, les modifications dans la structure du système productif ne sont pas prises en considération, notamment en ce qui concerne les degrés d'intégration de l'industrie et des services. C'est pour ces raisons que les recherches agrégées ont été graduellement abandonnées au profit d'analyses basées sur des essais de désagrégation. Ainsi, un certain nombre d'hypothèses rejetées sur la base de données agrégées pourront être acceptées sur des portions spécifiques du tertiaire, confirmant alors la nature non unitaire du phénomène.

\section{Les analyses désagrégées du secteur des services}

La plupart des recherches présentées récusent, à certains égards, la vision conventionnelle de l'avenir des économies développées. L'examen de vingt-cinq ans de modifications de l'emploi et des modèles de consommation au RoyaumeUni a révélé non pas l'émergence graduelle d'une économie de services, mais le contraire. Au lieu de trouver une hausse considérable de la consommation des services, on assiste à la baisse remarquable de leur consommation en valeur relative (Gershuny, 1978). Au lieu d'acheter des services, les ménages semblent acheter de façon croissante des biens durables qui leur permettent de produire des services pour eux-mêmes. Cela correspond à ce que J. Gershuny appelle 
«self-service economy». Une grande part de l'emploi tertiaire sur la période peut s'expliquer non par la consommation des services, mais par le besoin de l'activité manufacturière en techniciens, gestionnaires, vendeurs pour la production effective de biens de consommation. On a toutes les raisons de penser que cette tendance se poursuivra dans la mesure où elle est expliquée par deux processus économiques dont la direction ne peut pas varier: l'innovation technique et le renchérissement des coûts salariaux. On est donc loin de la thèse de la désindustrialisation des économies.

D'autres travaux corroborent ces résultats. La seule croissance relative des services semblerait être celle des services peu ou pas substituables à des biens comme, par exemple, les services médicaux et les assurances. L'explication semble résider dans une substitution continue au cours du temps de biens aux services, notamment de biens durables. Ainsi, un certain nombre de fonctions peuvent être remplies indifféremment par un bien ou un service et on peut remarquer que les biens fournissent également des services. Ceci étant, si la demande de services ne semble pas avoir le caractère massif qui lui est généralement attribué, il reste à démontrer pourquoi l'emploi tertiaire a crû de telle façon. Cela peut s'expliquer en partie par une mise en œuvre de politiques d'inspiration keynésienne ayant entraîné le gonflement des dépenses publiques, notamment en matière de couverture sociale et d'éducation, ainsi qu'en partie par une élévation des niveaux de vie (Vincent-Thomas, 1980). Les services du secteur public, tels que l'enseignement et la recherche, sont ceux qui croissent le plus rapidement; s'y ajoutent les services au producteur, essentiellement parce qu'une part plus importante des ressources est consacrée à l'ingénierie, la recherche-développement et à la direction et au développement de la firme (Noyelle, 1985).

\subsection{Les analyses des liens entre biens et services}

Partant de la constatation que le secteur des services n'a souvent été étudié qu'occasionnellement en profondeur et essentiellement pour ce qui concerne les services au consommateur, une monographie centrée sur les services au producteur a permis d'en révéler l'évolution (Greenfield, 1966). La division entre les biens et les services n'est pas précise et il est tentant d'appliquer aux services la vision dichotomique producteur-consommateur qui est appliquée aux biens, telle qu'elle a été avancée par S. Kuznets (1966). Ce dernier a en effet présenté un schéma de classification des biens en créant une double division de base entre les «producer goods" et les «consumer goods» comportant chacune trois subdivisions : périssable, semi-durable et durable. Pour H.I. Greenfield, ce schéma taxonomique peut être en grande partie appliqué aux services. Les biens destinés aux consommateurs sont ceux définis comme satisfaisant la demande 
finale, ceux destinés aux producteurs, comme ceux entrant dans la production intermédiaire. De même, les services au consommateur satisfont la demande finale et les services au producteur, la consommation intermédiaire. Mais cette classification de biens et services n'est pas définitive; bon nombre d'entre eux sont difficiles à classer et des décisions arbitraires doivent occasionnellement intervenir. Néanmoins, il est important de noter le parallélisme entre bien et service, et le fait que les services peuvent être analysés en des termes qui autrefois étaient exclusivement réservés aux biens. Partant de là, il devient possible de raisonner en termes de stocks, d'obsolescence, de financement. L'une des plus importantes applications de la durabilité des services se trouve dans l'analyse des fluctuations de la demande dans le cycle des affaires. H.I. Greenfield est le premier auteur à étudier de façon détaillée les services destinés au producteur. Son approche représente un premier pas vers une meilleure compréhension du rôle des services dans le processus économique, et il est intéressant de souligner le rôle du capital immatériel dans le processus de production. Il remarque d'ailleurs que, dès le début des années 50, des recherches avaient reconnu les améliorations dans les méthodes et le savoir-faire comme un type distinct de dépense en capital. Ses travaux marquent un pas important dans l'intégration des services dans l'industrie. Statistiquement, cette affirmation est confirmée dans la mesure où, depuis quelques dizaines d'années, on assiste à un accroissement marqué des «cols blancs » par rapport aux «cols bleus», particulièrement, dans le principal secteur produisant des biens dans l'économie, c'est-à-dire l'industrie. Le fait est, souligne H.I. Greenfield, que les travailleurs dans les services sont aussi importants dans le processus productif que ceux qui se consacrent à la fabrication.

D'autres travaux confirment les liens entre les biens et les services nés de complémentarités (Barcet, 1987), notamment de la demande (Stanback, 1979). Couplée à cette complémentarité de la demande, il y a possibilité de substitution de biens aux services quand les tendances de coûts et de prix des services sont défavorables. Une telle substitution restreint donc le changement brusque d'une économie de biens vers une économie de services. Les résultats de T.M. Stanback (1979) corroborent ceux de V.R. Fuchs (1977) qui établissaient que lorsque la production est mesurée en dollars constants, il y a très peu de variations dans la part respective des biens et des services depuis 1961 aux États-Unis ${ }^{10}$. Dans les travaux de T.M. Stanback, les activités communément décrites comme des services ont des caractéristiques différentes. Tout d'abord,

10. Selon Fuchs (1977) la répartition du Produit national brut américain par secteur d'activité n'a pratiquemment pas varié depuis 1961 en dollars constants, puisque les services représentent $51,3 \%$ en $1948,55 \%$ en 1961 et $56,3 \%$ en 1976 (on a respectivement pour l'industrie : $43 \%, 40,7 \%$ et $40,7 \%$ ). Toutefois, il faut noter que dans ces calculs, l'industrie inclut les transports, les communications et les services publics, ce qui peut introduire un biais dans l'analyse. 
il y a trois types de services: les services destinés au producteur, ceux destinés au consommateur et les services publics. En réalité, cette différenciation n'est pas parfaite dans la mesure où une même firme peut produire à la fois des services au producteur et au consommateur, comme elle peut produire des biens de production et des biens de consommation. Ce sont essentiellement les sources de la demande qui diffèrent et qui, par conséquent, justifient cette différenciation. De plus, certains services ont une forte intensité en travail, d'autres en capital, et il existe de grandes différences en ce qui concerne la taille des entreprises. Si l'on admet que la production de biens a pour résultat un produit matériel, stockable et transportable et que celle des services a pour résultat un output non stockable requérant habituellement une interaction directe avec le consommateur, cette distinction signifie que les biens peuvent être produits plus facilement que les services sous des conditions qui revêtent l'avantage d'une production standardisée et des économies d'échelle. Ceci résulte à la fois des différences dans la localisation des firmes et dans leur capacité à utiliser des modes de production à forte intensité capitalistique. Les services sont moins standardisés et les firmes qui les produisent tendent à être localisées près des marchés sur lesquels elles traitent, souvent dans des régions trop peuplées, ce qui n'est pas sans avoir de conséquences sur leurs coûts de production. Le problème soulevé par Stanback est de savoir comment définir les services en excluant les activités qui sont les plus proches des biens, c'est-à-dire avec une forte intensité capitalistique et des modes de production à grande échelle. En conséquence, la solution consisterait à restreindre la définition des services et exclure (comme dans les travaux de V.R. Fuchs) les transports, les communications et les services publics, activités alors reportées dans l'industrie. Ces remarques mettent en évidence les forts liens entre biens et services nés de complémentarités de la demande. Au niveau de la consommation, ces complémentarités sont largement issues de leur usage commun, en particulier, le besoin de distribuer et d'entretenir les biens. Au niveau de la production, elles sont de façon croissante le résultat de la complexité accrue de la gestion qui crée de nouvelles exigences et de l'utilisation croissante d'entreprises de services. Il ne faut pas oublier que couplée à cette complémentarité, il y a la possibilité toujours présente de la substitution de biens aux services qui restreint la possibilité du passage d'une économie de biens à une économie de services.

D'autres travaux ont contribué à la démonstration de l'interaction services-industrie, à partir d'une méthodologie originale (Momigliano et Siniscalco, 1980, 1982). Leurs hypothèses reposent sur le fait que, d'une part, la croissance relative et absolue de l'emploi tertiaire est due en grande partie à l'augmentation de l'intégration des services dans le système productif et que, d'autre part, le plus grand degré d'intégration peut être spécifiquement attribué à la croissance des services pour l'industrie et plus généralement à la production de biens (Siniscalco, 1985). Selon ces hypothèses, la croissance relative et 
absolue de l'emploi des services provient largement d'une utilisation accrue des activités classées dans le tertiaire, mais intégrées dans le système productif et dans l'industrie en particulier. Si elles sont acceptables, cela signifie bien que les changements en cours ne correspondent pas à une transition vers une économie de services ou vers une société postindustrielle.

F. Momigliano et D. Siniscalco rejoignent dans leur interprétation des services les auteurs précédents, mais ils présentent l'originalité de proposer une méthodologie basée sur les tableaux d'entrées-sorties (instrument déjà utilisé par Carter, 1970) et conduisant à une nouvelle désagrégation des services fondée sur la destination finale au sein du système. Mais pour étudier des grandeurs comme les services destinés à l'industrie (c'est-à-dire le volume spécifique d'un service utilisé dans un produit spécifique), les tableaux d'entrées-sorties présentent des limites; et pour une analyse détaillée des processus productifs, ces auteurs proposent une autre méthode permettant de désagréger le système en le répartissant en sous-systèmes ou en secteurs verticalement intégrés selon les concepts introduits par P. Sraffa (1960) et L. Pasinetti $(1973,1981)$.

Leurs résultats (basés sur des tests ayant pour cadre l'économie italienne) indiquent que les services au système productif représentent une part considérable de l'emploi des services marchands (40\% en 1981) et que la croissance de l'emploi des services au système productif est beaucoup plus rapide que la croissance des services marchands, représentant ainsi une proportion élevée de la croissance de l'emploi dans les services marchands. De plus, les services à l'industrie constituent la composante la plus dynamique des services marchands, puisqu'ils croissent plus vite que les services au système productif (respectivement une augmentation de $95 \%$ et de $36 \%$ sur la période 1965-1981).

Les résultats de ces travaux montrent l'importance considérable et croissante des services au producteur, ce qui permet de valider l'hypothèse de l'intégration des services dans la production et donc de contredire formellement les explications de la croissance des services par la théorie des étapes ou celle de la désindustrialisation. Ainsi, bien que la production et l'emploi se déplacent progressivement vers les services, les économies avancées restent des économies de biens.

\subsection{La remise en cause des caractères spécifiques attribués aux services}

Si l'interaction entre les services et les biens ne semble faire aucun doute, il n'en reste pas moins que les services conservent des caractéristiques qui leur sont propres. Mais on peut toutefois tenter de remettre en question certaines spécificités qui leur sont trop vite et trop largement attribuées: immatérialité, 
intangibilité, forte valeur ajoutée, faible intensité en capital et faible productivité; ceci nous permettra d'appuyer la thèse de l'interaction entre les biens et les services et celle de leur similitude par rapport à plusieurs axes d'analyse. En effet, si l'on considère que les services contiennent une part importante de valeur ajoutée, se pose alors le problème de savoir si ce sont des activités sans amont. D'après les arguments présentés précédemment, ce n'est pas le cas pour bon nombre d'entre eux, ce qui rejoint la critique portée à l'égard de leur immatérialité. Le pourcentage de la valeur ajoutée ne constitue pas un critère absolu permettant de distinguer la production de biens et celle de services (Lengelle, 1966). L'idée que les services sont éphémères et sans substance parce que ce sont des biens immatériels apparaît comme une erreur pernicieuse (Hill, 1977). Par ailleurs, l'utilisation de la valeur ajoutée comme mesure de la production des services ne renseigne pas sur la véritable valeur pour l'utilisateur du service dispensé (St Geours, 1982).

À côté de l'importance de la valeur ajoutée, les services se voient généralement attribuer une faible intensité en capital. S'il est vrai que le tertiaire «traditionnel » possède un coefficient de capital relativement faible par rapport aux activités industrielles, il n'en est pas de même pour plusieurs activités de services «modernes »: la croissance de la part du tertiaire dans l'économie s'accompagne d'une croissance de sa consommation de capital. C'est particulièrement le cas de la santé, de l'éducation et des télécommunications. Ce critère d'intensité du capital met en évidence des disparités existant à l'intérieur de ce secteur, ainsi que le lien entre croissance de l'activité économique et croissance de l'investissement.

Enfin, les services se trouvent caractérisés par une faible productivité, ce qui peut alors apparaître comme étant une des causes majeures du ralentissement de la productivité globale de l'économie (Lorenzi, Pastre et Toledano, 1980; de Bandt, 1985), ou par une productivité présentant de considérables diversités selon les activités de services (Fabricant, 1972). Les activités tertiaires ne donnant pas lieu à la fabrication de produits, on ne peut donc pas utiliser le critère de productivité physique. Il faut donc utiliser la productivité en valeur au prix du marché ou au prix des facteurs. Le fait de recourir à la valeur tend toutefois à renforcer le caractère d'immatérialité et d'intangibilité propre à la plupart des services, ce qui n'apparaît pas très satisfaisant compte tenu de ce que nous avons tenté de démontrer précédemment. Le problème est donc de savoir comment mesurer l'output et la productivité d'un individu exerçant une activité tertiaire. L'objectif est alors de mesurer l'importance des facteurs qualitatifs dans l'appréciation des activités de services. Certes le progrès technique étant plus élevé dans l'industrie, c'est là une cause essentielle de l'augmentation relative du nombre d'emplois tertiaires. Ceci étant, pour certaines activités tertiaires la productivité croît plus rapidement - sinon plus - que pour l'ensemble de 
l'économie. La raison doit en être recherchée du côté de la demande. Dans des travaux particuliers sur la productivité du tertiaire, on remarque que la part de l'accroissement de la productivité due à la main-d'œuvre (amélioration des qualifications) est d'autant plus importante que le «produit» tertiaire est immédiatement consommé (Fuchs, 1968). La valeur d'usage du service ne dépend pas seulement de celui qui le procure, mais également de celui qui le reçoit ou le sollicite ou, bien souvent, du rapport entre ces deux agents. La qualité du service rendu dépend de l'interaction entre le prestataire et le client. Certains voient ainsi, dans l'industrialisation extrême du service, l'unique moyen d'améliorer la productivité du tertiaire (Eiglier et Langeard, 1979). La hausse de la croissance de la productivité notée dans les services ne serait pas différente de celle observée dans les activités produisant des biens (Kutscher et Mark, 1983) et l'hypothèse du transfert d'emploi dans les services comme responsable de la faible productivité ne serait pas fondée (Runyon, 1985). Toutefois, la caractéristique que l'on prête généralement à la productivité du tertiaire impose une limitation sévère pour faire des activités tertiaires un pilier de la croissance; il faut de fait garantir une croissance «autonome» de la demande de services d'autant plus importante que les gains de productivité n'apparaissent qu'au-delà d'un certain seuil de croissance (Petit, 1987). L'hypothèse retenue ici est fondée sur le développement des activités de services aux entreprises, dans la mesure où il semble possible de dégager des gains de productivité plus importants en raison surtout de la division du travail entre firmes.

\subsection{Services aux entreprises et dynamisme des PME: une mise en perspective}

La place des services devient stratégique dans l'évolution du système productif et leur développement n'a de signification que s'ils sont capables de le dynamiser. Le service devient de plus en plus l'autre face d'un bien dont l'utilisation suppose le développement du service.

La croissance du flux de consommation intermédiaire de services représente le phénomène le plus remarquable du fonctionnement des pays de l'OCDE au cours des vingt dernières années.

Les services rendus aux entreprises constituent la composante dynamique des activités de services: dans les pays de la Communauté européenne, leur taux annuel de croissance moyen a été de près de $15 \%$ par an de 1980 à 1985. Plus particulièrement, ce sont les services dits «informationnels », c'est-à-dire ceux qui participent directement au processus de production, qui ont connu les croissances les plus fortes (de Bandt et Petit, 1992). Ces services interviennent dans 
différents domaines et sont susceptibles d'aider les entreprises; ils permettent aux entreprises industrielles d'avoir accès à de nouvelles manières de faire.

L'imbrication entre industrie et services se manifeste sous deux formes: les emplois de services peuvent être situés soit à l'intérieur des entreprises, soit à l'extérieur (Bailly et Maillat, 1988). Si ce sont essentiellement les grandes entreprises industrielles qui internalisent leurs fonctions de services, les PME, elles, étant donné leur taille, satisfont une part importante de leurs besoins en services par un recours à l'extérieur afin d'améliorer leurs techniques de production et leurs méthodes de gestion, en raison de la séparation des fonctions de production et de décision.

Les PME et les PMI représentent une part majoritaire des activités économiques. Pour être efficaces dans le contexte actuel, elles ont besoin de conditions favorables pour pouvoir avoir accès à des marchés plus étendus et diversifiés, disposer de technologies nouvelles pour leurs activités de production et leurs modes d'organisation. Le recours aux services extérieurs apparaît donc comme un facteur clé de la compétitivité des PME. Leur dynamisme et leurs performances dépendent alors de l'efficacité de ces services externes. Cette remarque concerne les entreprises industrielles, mais aussi les entreprises de services: il y a alors un effet d'entraînement entre les entreprises de services.

Tout ceci traduit une tendance nouvelle de l'organisation du système productif, imbrication du tertiaire et de l'industrie. En fait, les services se trouvent en étroite relation avec les deux actes fondamentaux de l'économie marchande: la production et l'échange (Barcet et Bonamy, 1988).

Le développement des services rendus aux entreprises atteste une mutation du mode de production. Ce ne sont pas les services qui se développent au détriment des biens, mais il est clair que ce sont de nouvelles relations entre biens et services. Dans les économies actuelles caractérisées par le développement des services, l'acte complet de production ne s'achève que lorsqu'il y a eu maximisation de l'utilisation conjointe des produits et des services.

Le phénomène réel à observer est la progressive interpénétration de toutes les activités économiques. Ce que l'on appelle souvent la «nouvelle économie de service» ne correspond pas à l'économie tertiaire au sens traditionnel, mais est basée sur le fait que les fonctions de services dominent tout autre type d'activité économique. Les services deviennent des outils indispensables de production et ainsi, les biens ne sont utilisables que grâce aux fonctionnements des services (Giarini et Roulet, 1988).

Dès le début des années 80 , des travaux posaient l'hypothèse que l'un des éléments des stratégies de redéploiement des PMI dans la période actuelle était le recours à des services internes, mais aussi plus probablement externes 
(Barcet et Bonamy, 1982). Les PME peuvent difficilement internaliser des activités de services, en raison de coûts trop lourds. Au niveau régional, par exemple, le développement d'activités de services permet aux PME de s'approvisionner sur un marché local en externalisant ces fonctions et ainsi de se maintenir dans leurs régions.

Il paraît souhaitable d'encourager les entreprises de services, trop souvent implantées dans les métropoles, à se localiser dans des zones où les PME sont dynamiques ou de favoriser l'apparition d'entreprises de services lorsque de véritables marchés semblent possibles. Il y a alors un effet d'entraînement entre les PME/PMI qui externalisent leurs fonctions de services et les entreprises de services.

Les développements précédents ont permis de mettre en évidence les limites essentielles des thèses dites de la tertiarisation et de la désindustrialisation. D'une part, en effet, leur fondement théorique principal, c'est-à-dire le découpage trisectoriel de l'économie, ne semble pas présenter le degré de généralité qu'on lui attribue souvent. D'autre part, la distinction entre secteurs secondaire et tertiaire est de plus en plus sujette à interrogation, en raison du phénomène constaté de croissance des services à l'industrie. Ces limites rendent nécessaires le recours à un raisonnement différent qui relativise l'importance de la dissociation entre biens et services. Il convient d'introduire de nouveaux fractionnements de l'économie. Cette perspective s'avère centrale du point de vue de l'économie industrielle. Moins que jamais, celle-ci ne saurait cantonner son objet au secteur secondaire. Elle doit nécessairement étendre ses investigations aux autres secteurs et, en particulier, à une des composantes les plus dynamiques du secteur des services, à savoir les services à l'industrie.

\section{Bibliographie}

AANESTAD, J.M. (1987), «Measurement problems of the service sector », Business Economics, vol. XXII, $\mathrm{n}^{\circ} 2$, avril.

Bacon, R. et 'W. Eltis (1976), Britain's Economic Problem: Too Few Producers, Londres, MacMillan.

BAILly, A. et D. MAILlat (1988), Le secteur tertiaire en question, Neuchâtel, Éditions régionales européennes et Paris, Économica.

BARCET, A. (1987), «Tertiarisation de l'économie, marché du travail et emploi», Économie et Humanisme, $\mathrm{n}^{\circ} 295$, mai-juin.

BARCET, A. et J. BonAMY (1982), «Développement des services et redéploiement des PMI », Rencontre de recherche: Tertiarisation et PMI, Lyon, février. 
BARCET, A. et J. BONAMY (1988), «Services et transformation des modes de production ", Revue d'Économie Industrielle, $\mathrm{n}^{\circ} 43,1^{\mathrm{er}}$ trimestre.

BAUER, P.T. et B.S. YAMEY (1951), «Economic progress and occupational distribution », Economic Journal, décembre.

BAUER, P.T. et B.S. YAMEY (1954), «Further notes on economic progress and occupational distribution », Economic Journal, mars.

BELl, D. (1973), The Coming of the Post-Industrial Society, Londres, Heinemann.

Brender, A., A. Chevalier et J. PiSANI-FERry (1985), «États-Unis : croissance, crise et changement technique dans l'économie tertiaire», Économie Prospective Internationale, Paris, CEPII, Documentation française.

CAIRnCross, A.K. (1978), « What is de-industrialisation ? » dans F. Blackbaby (dir.), De-industrialisation, Londres, Heinemann.

CARTER, A.P. (1970), Structural Change in the American Economy, Cambridge, Massachusetts, Harvard University Press.

Clark, C. (1940), The Conditions of Economic Progress, Londres, MacMillan.

DE BANDT, J. et al. (1985), «La productivité dans les activités de services», Cahiers Services $n^{\circ} 1$, juin, Ministère de la Recherche et de la Technologie.

DE BANDT, J. et P. PETIT (1992), « Compétitivité : la place des rapports industrie/services », Séminaire international : Le devenir industriel de la France: coopérer, 7,8 et 9 septembre.

Dockes, P. (1969), L'espace dans la pensée économique du $16^{e}$ au $18^{e}$ siècle, Paris, Flammarion, Nouvelle Bibliothèque Scientifique.

EIGLIER, P. et E. LANGEARD (1979), «Eléments pour une théorie des services : application à l'entreprise», Cahier de recherche $n^{\circ} 142$, IAE Aix-en-Provence, janvier.

FABRICANT, S. (1972), «Productivity in the tertiary sector», National Bureau Report, numéro spécial, $\mathrm{n}^{\circ} 10$, aout.

FISHER, A.G.B. (1933), «Capital and the growth of knowledge», Economic Journal, septembre.

FISHER, A.G.B. (1935), The Clash of Progress and Security, Londres, MacMillan.

FISHER, A.G.B. (1939), «Production, primary, secondary and tertiary », Economic Record, juin.

FISHER, A.G.B. (1945), Economic Progress and Social Security, Londres, MacMillan.

FISHER, A.G.B. (1946), «Tertiary production as a postwar international economic problem », Review of Economic Statistics, août.

FISHER, A.G.B. (1952), «A note on tertiary production », Economic Journal, décembre. 
FISHER, A.G.B. (1954), «Tertiary production: a postcript », Economic Journal, septembre.

FOURASTIÉ, J. (1950), Le grand espoir du $20^{e}$ siècle, Paris, PUF.

FUCHS, V.R. (1965), "The growing importance of the services industries», The Journal of Business of the University of Chicago, XXXVIII, octobre.

FUCHS, V.R. (1968), The Service Economy, NBER, New York, Columbia University Press.

FUCHS, V.R. (1977), «The service industries and US economic growth since World War II », NBER, WP n 211.

GIARINI, O. et J.R. RouLET (1988), « Repenser l'offre: la relance des activités productives par l'économie de service», sous la direction d'O. Giarini et J.R. Roulet, L'Europe face à la nouvelle économie de service, Publications de l'Institut universitaire d'études européennes, Genève; Paris, Presses universitaires de France.

GERSHUNY, J. (1978), After Industrial Society ? The Emerging Self-Service Economy, Londres, MacMillan.

GREENFIELD, H.I. (1966), Manpower and the Growth of Producer Services, New York et Londres, Columbia University Press.

HILL, T.P. (1977), « On goods and services », Review of Income and Wealth, $n^{\circ} 1$, mars.

JACCARD, M. (1944), La mobilité de la main-d'cuvre et les problèmes du chômage et de la pénurie de travailleurs, Lausanne, Collection de la Nouvelle École de Lausanne.

JACCARD, P. (1953), «Les thèses de Petty, Fisher, Clark, Fourastié sur les conditions du progrès économique et social », Revue économique et sociale, Lausanne, octobre.

KATOUZiAN, M.A. (1970), «The development of the service sector : a new approach », Oxford Economic Papers, vol. 22, $\mathrm{n}^{\circ} 3$, novembre.

KUTSCHER, R.E. et J.A. MARK (1983), «The service producing sector: some common perceptions reviewed », Monthly Labor Review, avril.

KUZNETS, S. (1966), Modern Economic Growth Rate Structure and Spread, New Haven, Yale University Press.

LENGELle, M. (1966), La révolution tertiaire, Paris, Éd. Génin.

Lorenzi, H., O. Pastre et J. Toledano (1980), La crise du $20^{e}$ siècle, Paris, Économica.

Marshall, A. (1979), Principle of Economics, $8^{e}$ édition, Londres, MacMillan.

Momigliano, F. et D. SinisCalco (1980), «Terziario totale e terziaro per il systemo produttivo », Economia e politica industriale, $\mathrm{n}^{\circ} 25$. 
Momigliano, F. ET D. Siniscalco (1982), « The growth of service employment: a reappraisal », Banca Nazionale del Lavoro, $\mathrm{n}^{\circ} 142$, septembre.

NoYelLE, T. (1985), «Dualisme dans les services: le cas des États-Unis », dans J. de Bandt (dir.), Les services dans les sociétés industrielles, Paris, Économica.

OJALA, E.M. (1952), Agriculture and Economic Progress, Londres, Oxford University Press, Geoffrey Cumberlege.

PASINETTI, L.L. (1973), «The notion of vertical integration in economic analysis », Metroeconomica, XXV.

PASINETTI, L.L. (1981), Structural Change and Economic Progress, Cambridge, Cambridge University Press.

PETIT, P. (1986), Slow Growth and the Service Economy, Londres, Frances Pinter Publishers.

PETIT, P. (1987), «Tertiarisation, croissance et emploi: quelques nouvelles logiques », Communication au colloque des communautés européennes, Transformation de l'emploi: nouvelles formes, nouveaux domaines, mai.

Rostow, W.W. (1960), The Stages of Economic Growth, Cambridge, Cambridge University Press.

RUNYON, H. (1985), «The service industries: employment, productivity and inflation », Business Economics, vol. $\mathrm{XX}, \mathrm{n}^{\circ} 1$, janvier.

St Geours, J. (1982), «Le développement du secteur tertiaire peut-il sauver la croissance et l'emploi ?», Revue d'Économie Politique, $\mathrm{n}^{\circ} 3$.

SingelmanN, J. (1978), From Agriculture to Services - The Transformation of Industrial Employment, Beverly Hills, Londres, Sage Library of Social Research, vol. 69.

SinisCalCO, D. (1985), «Emplois dans le secteur des services et changement structurel », dans J. de Bandt (dir.), Les services dans les sociétés industrielles, Paris, Économica.

SMITH, A.D. (1972), The Measurement and Interpretation of Service Output Changes, Londres, National Economic Development Office, mars.

SRAFFA, P. (1960), Production of Commodities by Mean of Commodities, Paris, Dunod, 1970 (traduction française par S. Latouche).

StANBAK, JR., T.M. (1979), Understanding the Service Economy, Employment, Productivity, Location, Baltimore et Londres, The John Hopkins University Press.

STIGLER, G.J. (1956), Trends in Employment in the Service Economy, NBER, Princeton, Princeton University Press.

TOURAINE, A. (1969), La société post-industrielle, Paris, Denoël. 
TRIANTIS, S.G. (1953), «Economic progress, occupational redistribution and international terms of trade», Economic Journal, septembre.

VERnIERES, M. (1985), L'emploi du tertiaire, Paris, Économica.

VINCENT-ThOMAS, E. (1980), «Les incertitudes du tertiaire», Critiques de l'économie politique, $\mathrm{n}^{\circ} 12$, juillet-septembre.

WOLFE, M. (1955), «The concept of economic sectors», Quarterly Journal of Economics, LXIX. 